Meta

Journal des traducteurs

Translators' Journal

\title{
Norme de communication et traduction de textes techniques
}

\section{Anne-Marie Loffler-Laurian}

Volume 29, numéro 2, juin 1984

URI : https://id.erudit.org/iderudit/003419ar

DOI : https://doi.org/10.7202/003419ar

Aller au sommaire du numéro

Éditeur(s)

Les Presses de l'Université de Montréal

ISSN

0026-0452 (imprimé)

1492-1421 (numérique)

Découvrir la revue

Citer cet article

Loffler-Laurian, A.-M. (1984). Norme de communication et traduction de textes techniques. Meta, 29(2), 175-181. https://doi.org/10.7202/003419ar d'utilisation que vous pouvez consulter en ligne.

https://apropos.erudit.org/fr/usagers/politique-dutilisation/ 


\section{NORME DE COMMUNICATION ET TRADUCTION DE TEXTES TECHNIQUES*}

Dans cet exposé, je me propose d'essayer de préciser en quoi la notion de norme intéresse la traduction technique, c'est-à-dire la traduction de discours de spécialité, que cette spécialité soit technologique ou de type "sciences humaines ou sociales ". Il s'agit donc de voir quelle définition de la norme pourrait être adoptée de façon que cette notion ne soit plus une vue de l'esprit mais un concept opératoire.

\section{QU'EST-CE QUE LA NORME?}

Toute production linguistique est subordonnée à une idée plus ou moins précise, plus ou moins consciente, plus ou moins explicitée, de l'existence d'une norme. Même ceux qui admettent aisément qu'il existe non pas une norme mais des normes différentes selon les situations de communication, ne sont pas toujours exactement au fait de ce qui se cache sous ce mot. En première approximation, il est toujours possible de parler de modèle. Il est des modèles linguistiques comme des modèles de comportement : les productions sont des imitations, des tentatives de calque, des reproductions d'attitudes ou de productions déjà réalisées, déjà testées, déjà réussies. La norme se situe quelque part dans ce modèle de réussite. Mais elle n'en reste pas moins modèle, ce qui signifie qu'en tentant de la respecter, on implique son non-respect. La norme semble être cet inaccessible abstrait et idéel - idéal. Cela explique que l'on ait pu discuter si longuement sur cette notion, sans arriver à aucun accord. Chacun parle en fonction de lui-même après avoir intériorisé, conceptualisé et explicité des données acquises dans la jeune enfance. J'aimerais proposer ici, après avoir évoqué les grandes tendances des idées classiques sur la norme, une solution pour en finir avec les idées floues - cette solution se fondera sur des situations de communication particulières et des traitements de discours particuliers. Un modèle réduit mais fonctionnant vaut parfois mieux qu'une théorie vaste et inopérante.

\section{a. norme et usage}

Une longue tradition linguistique oppose la norme à l'usage. Cette opposition est fondée sur l'opposition parallèle entre langue et discours. Le premier terme désigne un ensemble de virtualités, le second s'appuie sur des formulations effectivement réalisées. Cette idée d'une langue fonctionnant comme une entité indépendante - dont les discours ne seraient que des manifestations épisodiques et parcellaires - a permis d'établir un réseau de règles auxquelles les discours devraient obéir. Dans cette conception de la norme en langue, ce sont les usages en discours qui doivent se conformer, s'adapter aux règles. (Et ce n'est pas l'observation des réalités de langage performé qui fournit la matière à la description des lois du fonctionnement.)

* Communication : colloque de linguistique contrastive français-hongrois, PECS (Hongrie), novembre 1982. 
Qui dit règle évoque contrainte, obligation, voire rétorsion. Qui dit règle implique du même coup exception, dérogation, différence, c'est-à-dire, en un mot, liberté utilisée. Or toute langue, du seul fait qu'elle est une production humaine, porte en soit une manifestation de liberté. Tout discours est personnel parce que, quelque part, il ne colle pas parfaitement au modèle.

Liberté, personnalité, ce sont les voies par lesquelles le discours s'éloigne de cette idée vague de la norme, et par où, insensiblement se glissent les incorrections, les malformations, les fautes, bref tout ce qui concourt à cette plus ou moins lente dégradation de la langue que les amateurs du modèle fixe déplorent si souvent.

\section{b. attitude normative}

L'attitude normative apparaît lorsque des errements linguistiques ont été constatés et jugés répréhensibles ou nuisibles. Nuisibles à quoi ? À la beauté, à l'harmonie, à l'homogénéité de la langue par exemple. Répréhensible par rapport à quoi ? Par rapport aux règles édictées par la norme, par rapport à l'idée que l'on a du beau, du bon, du net.

Cette attitude implique une idée, ou plutôt une image du Bon et du Mauvais. I] faut, bien entendu que le Bon gagne, et pour cela il faut que la Loi arrive à s'imposer (une loi de règle et non d'usage, bien sûr).

La notion de faute est directement issue de cet avatar de la lutte entre le Bien et le Mal. La faute, y compris celle que l'on trouve sur les copies d'élèves - orthographe, grammaire, "faux-sens " ou " contresens ", etc. - est une incarnation non pas d'une incompréhension ou d'un défaut d'explication, mais d'un manquement à la loi. Elle mérite sanction.

L'attitude exploratrice ou observatrice qui consiste à décrire ce qui se passe dans les comportements linguistiques, qu'ils soient humains ou mécaniques, ne connaît pas la faute, mais seulement l'erreur. Ce terme, plus neutre, permet de constater un écart que l'on pourra ensuite mesurer par rapport à un étalon. La déviation, comme l'écart, est une donnée mesurable, comme le kilométrage d'une route que l'on a empruntée (ou prise ?) à la place d'une autre. Bien que les déviations soient en général installées de façon temporaire par les Ponts et Chaussées, il se peut que des automobilistes avisés y découvrent des raccourcis ou des échappatoires bien utiles dans certaines circonstances.

Comment cette attitude se manifeste-t-elle en traduction ? Où est le modèle qu'il faut imiter sous peine d'être sanctionné ? Dans l'enseignement, le manuel offre des modèles et des règles ; les étudiants ou les élèves de langues étrangères doivent $s^{\prime} y$ conformer. Le professeur apparaît en général comme l'incarnation de ce modèle. En fait, en classe de langue, dans l'enseignement secondaire, un autre modèle - redoutable s'il en fût - vient s'y greffer : c'est l'assistant de langue ${ }^{1}$, celui qui par définition connaît sa langue puisqu'il est natif et qu'il a fait des études supérieures, mais celui également qui est le fruit des tendances évolutives de sa langue et qui n'en manifeste qu'un état précaire et bien délimité géographiquement et temporellement. En traduction faudra-t-il imiter un manuel, un professeur, un natif ?

Bien souvent le natif fournit le critère de la correction (substantif correspondant à l'adjectif "correct", comme "correctness", et non nominalisation du verbe " corriger "). Pour bien traduire un discours, il faut l'exprimer comme un natif de la langue cible l'aurait fait. En respectant les niveaux (élaboré ou recherché, familier ou vulgaire, etc.), les mentalités, etc. Si c'est un idéal que l'on peut effectivement chercher à atteindre, c'est aussi, de fait, une gageure. Est-il possible d'organiser sa pensée ou d'avoir les réactions affectives de quelqu'un qui a été élevé dans un milieu différent, avec un

1. Cf. article de J.M. LAURIAN, dans Contrastes, no 4-5, novembre 1982. 
poids du passé différent ; histoire collective ou individuelle, personnalité propre fruit d'une éducation et d'un héritage, font qu'un non-natif ne peut coller au natif, il ne peut que s'en approcher ${ }^{2}$. C'est ce que les traducteurs les meilleurs arrivent à faire.

D'où le problème d'identification lié à la norme linguistique en traduction. Traduire c'est entrer dans la peau d'un étranger. Qui le veut ou le peut réellement ? Certains parviennent à en donner l'illusion, c'est du bon théâtre, et c'est tout ce qu'on demande.

Le traducteur est un acteur dont la norme consiste non seulement à épouser les règles du jeu de sa parole seconde mais également à jouer le personnage du locuteur premier.

La communication n'est pas de son ressort, c'est l'objectif du locuteur — et du récepteur éventuellement. Lui, il permet au discours de changer de costume. Le respect de la norme pour lui est dans le bon choix du costume.

\section{c. activité normalisatrice}

En particulier dans les domaines techniques, il est important que les mots soient univoques et qu'ils aient les mêmes référents pour tous les locuteurs, quelles que soient les situations de communication ou les types de textes. On est là tout spécialement dans le domaine du lexique, qui devient terminologie. Pour ce qui est des objets "importés ", qu'il s'agisse d'objets matériels ou non matériels, il est essentiel qu'il ne puisse y avoir d'ambiguité sur la réalité désignée. Une activité normalisatrice se met alors en place pour codifier les emplois.

De fait, cette activité s'étend non seulement au contrôle et à la création des vocables désignant des réalités nouvelles, mais également au jugement sur un certain nombre de vocables de domaines de spécialités. Ainsi des commissions ministérielles de terminologie ${ }^{3}$ ont-elles pu être créées afin de veiller à ce que des termes français existent et soient utilisés (obligatoirement) lorsque cela était possible. Dans le domaine de l'audiovisuel, on a ainsi remplacé "scriptgirl " par "scripte ", lui donnant une terminaison française, et "perchman" par "perchiste" selon le même processus de francisation du suffixe - si ce n'est qu'ici il ne s'agissait pas à l'origine d'un mot suffixé mais d'un mot composé ! Enfin, dans la mesure où un contrôle sur ce qui apparaît sur le petit écran est possible, ces termes ont pu être imposés. Et bien d'autres encore, dans d'autres domaines aussi. L'ensemble de six vocabulaires a été publié au Journal officiel. Par ailleurs l'AFNOR a pour rôle de normaliser non seulement les produits industriels mais aussi leurs désignations. De plus, elle fournit des fiches terminologiques fort utiles.

Enfin, il faut bien reconnaître que cette activité normalisatrice concerne davantage les administrateurs et les producteurs, les traducteurs parfois, mais les linguistes à proprement parler beaucoup moins souvent. Par contre l'usage qui résulte de ces normalisations intéresse de près les linguistes qui cherchent à décrire le langage technique français actuel.

\section{TRADUCTION DE TEXTES TECHNIQUES ET NORME}

Si la norme est déjà tellement malaisée à définir dans les champs dits de banalité ou de quotidienneté, qu'en est-il dans les domaines spécialisés ? À première vue, on pourrait penser que plus on restreint le domaine d'application, plus le langage devient aisé à circonscrire, ce qui signifierait qu'il existe une norme simple, toujours utilisable, pourvu que l'on sache à quel niveau de discours et à quel type de contenu on se tient. En

2. Il s'agit ici à la fois de thème et de version, de compréhension et de production.

3. Les résultats de certains travaux ont été publiés au Journal officiel sous le titre " Enrichissement du vocabulaire " mais d'autres sont encore en cours. 
discours monolingue, c'est peut-être vrai, mais en traduction, les problèmes apparaissent rapidement.

a. le cas des traductions au sein de la commission des communautés européennes

Notre analyse de la notion de norme qui suit se fonde sur l'étude d'un certain nombre de traductions produites à Luxembourg dans le cadre de la Commission des communautés européennes, et plus particulièrement de traductions automatiques produites par le système Systran qui y est utilisé.

Pour situer un peu ces traductions, disons qu'elles traitent des domaines aussi divers que l'exploitation des mines de charbon, les conditions de sécurité dans ces mines, le droit de la mer, les centres de documentation, l'informatique et les bases de données, les comptes rendus et procès-verbaux des réunions de spécialistes des pays membres sur tel ou tel sujet, etc. Tous les textes sont traduits vers toutes les langues de la Communauté, mais la machine ne traite que le français, l'anglais et l'italien à Luxembourg. Les textes qui sortent de l'ordinateur sont des " traductions brutes" qui sont ensuite corrigées, - on dit plutôt "postéditées" - par des "postéditeurs " qui sont des traducteurs de formation et qui acceptent de travailler sur des sorties d'ordinateur.

Les traductions brutes contiennent des erreurs, en nombre plus ou moins grand (selon le degré de technicité du vocabulaire, selon le degré de complexité de la syntaxe, etc.). La correction de ces erreurs s'appuie sur une idée de la norme qui est constituée essentiellement de deux éléments :

- l'idée que le postéditeur a de la bonne langue, de la belle langue;

- la connaissance qu'il a des habitudes d'expression des locuteurs de tel ou tel domaine dans telle ou telle situation.

b. problématique des buts de la postédition

Deux tendances coexistent, s'affrontent ou se complètent selon les circonstances. Elles sont fondées sur les deux éléments cités ci-dessus. Ce sont :

- le désir de fournir au "client » un beau texte, c'est-à-dire une rédaction aussi élégante que possible, d'un style choisi et si possible d'un niveau élevé (il arrive que le traducteur "corrige» l'original pour lui insuffler quelque qualité stylistique);

- la nécessité de rapidité : c'est elle qui justifie l'utilisation de l'ordinateur (outre le prix de revient nettement moins élevé), liée au rôle tenu par le texte : document d'information destiné à fournir au lecteur une connaissance rapide et précise sur un sujet par exemple lorsqu'il s'agit de documents préparatoires à une réunion.

On peut considérer que l'exactitude est un minimum de toute traduction. Reste le conflit ou la complémentarité de la compréhensibilité et du style. On pourrait objecter qu'un texte incompréhensible est forcément inexact ; oui, mais un texte peut être exact du point de vue des données et cependant difficilement compréhensible pour des raisons de style.

Mais qu'est-ce que le style ? Est-ce quelque chose que l'on surajoute au contenu pour décorer, pardon, pour embellir l'expression, ou bien est-ce une composante intrinsèque de la formulation?

Si cette question était résolue, il n'y aurait plus qu'à édicter les règles minimales du style et à les respecter pour faire de bonnes traductions ou de bonnes postéditions. Et le travail des postéditeurs serait grandement accéléré. Mais la réponse à la question demande encore quelques recherches...

Voyons, sur quelques exemples particuliers comment on peut proposer de décrire l'ensemble norme, style et message. 


\section{c. les formes verbales}

Pour ce qui concerne le temps des verbes, si la machine donne un présent anglais pour un présent français, et vice versa, ou un passé français (passé composé ou imparfait) pour un passé anglais preterit, cela ne change par la compréhensibilité du texte, et l'exactitude théorique ${ }^{4}$ n'est pas mise en cause. Cependant, il y a « inexactitude " ou plutôt non respect des habitudes de la langue cible. En effet, dans les comptes rendus de réunions en particulier, il est d'usage d'employer le présent en français et le passé en anglais. Le postéditeur corrige donc la traduction brute.

Exemples :

$\mathrm{E} \rightarrow \mathrm{F} \quad$ he stated $\rightarrow$ il a déclaré $\rightarrow$ il déclare

still remained open $\rightarrow$ restait toujours ouverte

was giving cause for concern $\rightarrow$ provoquait les soucis $\rightarrow$ est une source de préoccupation

$\mathbf{F} \rightarrow \mathrm{E} \quad$ souhaite la bienvenue $\rightarrow$ welcomes $\rightarrow$ welcomed

est adopté $\rightarrow$ is adopted $\rightarrow$ was adopted

cette liste est nécessaire $\rightarrow$ this list is necessary $\rightarrow$ this list was necessary le registre des véhicules comprend un code $\rightarrow$ the register of the vehicles comprehends a code $\rightarrow$ the vehicle register included a code

Un autre exemple est celui de la présentation personnelle ou non personnelle de l'énonciation. Il existe une hiérarchie de personnalisation / dépersonnalisation qui va de l'énoncé personnel (avec références individuelles : $j e, t u, i l$, etc.) à l'énoncé en voie de dépersonnalisation (par on) et à l'énoncé dépersonnalisé (par il + être/ + adjectif + de par exemple), et enfin à l'énoncé que j'appelle non personnel dans le sens où l'agent n'y figure pas fortement lié au verbe (c'est ce que donne la "voix inverse "). La nominalisation est le stade ultime de la dépersonnalisation, mais ne nous intéresse pas ici puisque nous parlons du verbe et qu'elle en est un avatar discutable.

Exemples :

$\mathrm{E} \rightarrow \mathrm{F} \quad$ it was probably best to $\rightarrow$ il était probablement meilleur de $\rightarrow$ la meilleure solution était probablement

when there was a leakage of fuel $\rightarrow$ quand il y avait une fuite de combustible $\rightarrow$ une fuite de combustible présente un risque sérieux

Dans le sens anglais $\rightarrow$ français, la correction fait passer d'une forme moins personnelle à une forme plus personnelle du verbe. Dans le sens français $\rightarrow$ anglais, c'est l'inverse : on augmente la dépersonnalisation :

$\mathrm{F} \rightarrow \mathrm{E}$ dans une première phase il serait demandé aux traducteurs de $\rightarrow$ in a first phase, it would be asked the translators to $\rightarrow$ the translators would be asked in the first phase to

on peut choisir ces textes au hasard $\rightarrow$ one can choose these texts to the chance $\rightarrow$ these texts can be chosen randomly

on n'échappera donc pas à une expérimentation $\rightarrow$ one will not escape thus from a thorough experimenting $\rightarrow$ thorough experimenting will thus be essential

la recherche se propose de $\rightarrow$ the research proposes to $\rightarrow$ it is intended to

Forme en $i l$, forme en on, forme dite pronominale en se, ce sont trois variations françaises sur le thème de la dépersonnalisation de l'énoncé verbal, en face desquelles le postéditeur préfère en anglais une forme personnalisée.

Il est évident que ces transformations de la part du postéditeur ne peuvent être décrites comme des constantes absolues. Ce sont des tendances majoritaires plutôt. Elles

4. En général, c'est-à-dire si le temps verbal n'est pas l'objet de l'information. 
existent et dénotent des choix qui sont non pas au niveau de la langue, non pas non plus au niveau du correct ou de l'incorrect, et à peine du domaine du style au sens de l'élégance. Les choix sont stylistiques dans la mesure où choix il y avait. Mais leur objectif n'est pas une certaine perfection sinon une meilleure adéquation aux usages du locuteur natif qui aurait eu à régler le même texte en sa langue.

C'est la connaissance des usages et leur respect qui motivent les changements effectués par le postéditeur. Et pour lui, il apparaît que la norme est dans ce respect de l'usage. S'il ne respectait pas les formes habituelles des locuteurs de chaque langue, les lecteurs, bien que comprenant la signification du texte, et bien que se trouvant devant des textes corrects du point de vue de la grammaire, de la morphologie, du vocabulaire, etc., seraient troublés. C'est ce dérangement qu'il parvient à éviter en modifiant certaines traductions. Trouble, anormalité, dérangement, que de termes évoquant la sortie psychique hors du commun!

La norme, en traduction technique, d'après les postéditions de traductions automatiques, peut se redéfinir comme ce qui est d'usage le plus courant, le plus commun, le plus ordinaire. Est hors-norme ce qui détonne. Le meilleur style étant celui qui ne se voit pas, et c'est la norme.

\section{d. les lexies fixées et les sigles}

Les lexies fixées et les sigles sont des cas particuliers de vocabulaire et des cas particuliers de noms propres souvent. Si la question générale de la traduction des noms propres (est-elle possible? est-elle souhaitable ?) peut-être posée, y compris dans le cadre des questions relatives à la norme, à Luxembourg tous les sigles ont leur équivalent dans chacune des langues européennes et cet équivalent doit obligatoirement être respecté sous peine de rendre l'objet désigné méconnaissable.

En général, les sigles figurent dans le dictionnaire de la machine, et il n'y a pas de réel problème : il n'y a que des lacunes qui sont comblées dès la première apparition d'un sigle.

Cependant, parfois, il se passe des choses amusantes. Ainsi par exemple le français B.D. est passé en anglais en B.D., probablement parce que ce n'est pas un sigle conventionnel. Comme la lexie ainsi abréviée est exprimée dans l'ordre inverse en anglais et en français, le postéditeur a rétabli la forme complète : data base. Un autre exemple, dans le sens anglais $\rightarrow$ français, de sigle non "compris " par la machine est celui de $M E$ $D L A R S$, écrit en majuscules sans point entre les lettres. La machine a pris le nom commun nèfles pour le traduire, et le postéditeur a rétabli $M E D L A R S$ dans le texte français.

Dans des lexies plus complexes, la lexie doit être traitée globalement. Ainsi, European Coking Industry, qui peut être traduit " correctement » par industrie de cokéfaction européenne, sera modifié en industrie européenne de coke qui est la lexie complexe en usage pour désigner couramment cette réalité.

Dans certains cas, la lexie complexe est équivalente à un nom propre et devrait être traitée comme telle. Exemples :

le Comité intérimaire $\rightarrow$ the temporary committee $\rightarrow$ the interim committee

the Canadian Secretary of State's Technology Division $\rightarrow$ secrétaire canadienne de la division de technologie $\rightarrow$ la division de technologie du Secrétariat d'État canadien

Là, l'éloignement de la lexie équivalente de la langue cible rend l'expression totalement erronée. Il y a une gradation de la simple non-correspondance à la structure agrammaticale d'une lexie complexe. Ici la norme fonctionne comme en terminologie : à une réalité colle un terme, et tout autre terme est faux. 
e. verbaliser ou substantiver?

Si l'une des langues a une préférence pour des formulations substantivales par opposition à la préférence de l'autre langue pour des énoncés verbaux, cela ne signifie pas toujours que les traductions choisissant verbe ou substantif en contradiction avec la tendance des usagers de la langue soient erronées. Mais force est de constater qu'une norme s'installe rapidement, dictant le choix de l'un ou de l'autre en manière de contrainte.

Ex.emples :

minimising $\rightarrow$ réduisant à un minimum $\rightarrow$ diminution

to establish $\rightarrow$ pour établir $\rightarrow$ établissement

to nominate $\rightarrow$ pour nommer $\rightarrow$ désignation

Parfois la nominalisation semble introduire une simplification de l'expression. At-on alors un gain de style ? Ce gain, s'il y a, peut-il être érigé en norme?

Exemples :

where it is unlikely to be affected by fire $\rightarrow$ où il est peu susceptible d'être affecté par le feu $\rightarrow$ à l'abri du feu

/suite du précédent/ or to be hit by material projected $\rightarrow$ ou être frappé par le matériel projeté $\rightarrow$ hors d'atteinte des projectiles

\section{POUR CONCLURE}

La notion de norme porte en elle-même ses corollaires de faute - ou d'erreur d'écart - ou de déviance - , de dérogement - ou d'innovation. En admettant que la norme ait été revue à la lumière des notions de différence, de déviation, de niveau, de registre, il faut encore la revoir en fonction des situations de communication dans leur ensemble. À notre connaissance aucune théorie n'a encore structuré le monde des relations norme(s) - situations. La communication implique des relations affectives, émotives, intellectuelles, hiérarchiques, professionnelles, scientifiques, et bien d'autres encore. Celles-ci devraient être prises en compte par une description complète des " usages normés " dans différentes situations de communication.

Mais ce qui nous semble ici le plus important à souligner c'est l'aspect que prend la notion de norme à la lumière de la pratique de la traduction. Si les types de discours modifient l'attitude du traducteur face au texte (un texte littéraire n'est pas manipulé comme un texte technique), ils engagent aussi le modèle dans des voies diversifiées. Et l'on ne peut guère plus parler de modèle ; tout au plus pourra-t-on se référer à des fréquences d'emploi, à des habitudes expressives ou à des tics de formulation.

Un ensemble de contraintes est formé par tous ces usages que l'on peut répertorier bien qu'on ne puisse jamais être exhaustif. C'est cet ensemble, différent d'une situation de communication à une autre, différent d'un type de discours à un autre, et encore différent d'une langue à l'autre (à situation et type de discours sensiblement équivalent) que l'on peut appeler "norme de communication".

La lexie "norme de communication", plus concrète que la seule "norme », a l'avantage d'englober toutes les circonstances mentionnées ci-dessus. Elle ne se réfêre pas à un code écrit ou idéal mais aux habitudes langagières de telle ou telle communauté. Communauté professionnelle, scientifique, linguistique ... tous les déterminants 
se confondent dans un creuset d'où la notion de norme sort mieux adaptée aux réalités des communications pluridisciplinaires et multilingues du monde d'aujourd'hui.

ANNE-MARIE LOFFLER-LAURIAN 